\title{
A RELATION BETWEEN HIGHER WITT INDICES
}

\author{
NIKITA A. KARPENKO
}

\begin{abstract}
Let $\mathfrak{i}_{1}, \mathfrak{i}_{2}, \ldots, \mathfrak{i}_{\mathfrak{h}}$ be the higher Witt indices of an arbitrary non-degenerate quadratic form over a field of characteristic $\neq 2$ (where $\mathfrak{h}$ is the height of the form). We show that for any $q \in[1, \mathfrak{h}-1]$ one has

$$
v_{2}\left(\mathfrak{i}_{q}\right) \geq \min \left(v_{2}\left(\mathfrak{i}_{q+1}\right), \ldots, v_{2}\left(\mathfrak{i}_{\mathfrak{h}}\right)\right)-1
$$

where $v_{2}$ is the 2 -adic order. Besides we show that

$$
v_{2}\left(\mathfrak{i}_{q}\right) \leq \max \left(v_{2}\left(\mathfrak{i}_{q+1}\right), \ldots, v_{2}\left(\mathfrak{i}_{\mathfrak{h}}\right)\right)
$$

provided that $\mathfrak{i}_{q}+2\left(\mathfrak{i}_{q+1}+\cdots+\mathfrak{i}_{\mathfrak{h}}\right)$ is not a power of 2 .

These inequalities are applied in [3] to the problem of determination of the smallest possible height of an anisotropic quadratic form of any given dimension. The first inequality formally implies Vishik's conjecture on $\operatorname{dim} I^{n}$ proved previously in [6].

The method of the proof is that developed in [6]; it involves the Steenrod operations on the modulo 2 Chow groups of some direct powers of the projective quadric. It produces not only the above inequalities, but also some other relations between the higher Witt indices.
\end{abstract}

\section{Contents}

1. Introduction

2. Terminology, notation, and backgrounds 2

3. The upper bound 4

4. A trick 5

5. The lower bound 6

6. Holes in $I^{n} \quad 9$

References $\quad 9$

Date: June 11, 2004.

Key words and phrases. Quadratic forms, Witt indices, Chow groups, Steenrod operations, correspondences. 2000 Mathematical Subject Classifications: 11E04; 14C25.

Supported in part by the European Community's Human Potential Programme under contract HPRNCT-2002-00287, KTAGS. 


\section{INTRODUCTION}

We consider non-degenerate quadratic forms over fields of characteristic $\neq 2$ and establish the following result (the proof is given in $\S 3$ and $\S 5$; a definition of the higher Witt indices can be found in $\S 2)$ :

Theorem 1.1. Let $\mathfrak{i}_{1}, \mathfrak{i}_{2}, \ldots, \mathfrak{i}_{\mathfrak{h}}$ be the higher Witt indices of an arbitrary quadratic form. Then

$$
\text { (lower bound) } \quad v_{2}\left(\mathfrak{i}_{q}\right) \geq \min \left(v_{2}\left(\mathfrak{i}_{q+1}\right), \ldots, v_{2}\left(\mathfrak{i}_{\mathfrak{h}}\right)\right)-1
$$

for any $q \in[1, \mathfrak{h}-1]$, where $v_{2}$ is the 2 -adic order. Besides

$$
\text { (upper bound) } \quad v_{2}\left(\mathfrak{i}_{q}\right) \leq \max \left(v_{2}\left(\mathfrak{i}_{q+1}\right), \ldots, v_{2}\left(\mathfrak{i}_{\mathfrak{h}}\right)\right)
$$

provided that in the even-dimensional case the integer $\mathfrak{i}_{q}+2\left(\mathfrak{i}_{q+1}+\cdots+\mathfrak{i}_{\mathfrak{h}}\right)$ is not a power of 2 .

(Note that by [2] (see also [9, th. 7.3]) the upper bound inequality fails without the additional assumption which excludes the so-called case of maximal splitting.)

This Theorem is applied in [3] to the problem of determination of the smallest possible height of an anisotropic quadratic form of any given dimension. As shown in $\S 6$, the first inequality of this Theorem (together with Theorem 2.2) immediately implies Vishik's conjecture on $\operatorname{dim} I^{n}$, where $I$ is the fundamental ideal of the Witt ring of a field (see $\S 6$ ), proved previously in [6].

The method of the proof is that developed in [6]; it involves the Steenrod operations on the modulo 2 Chow groups of some direct powers of the projective quadric. It produces not only Theorem 1.1, but also some other relations between the higher Witt indices.

\section{Terminology, NOtATiOn, And BACKGROUnds}

We use the notation and terminology of [6]. In particular, $F$ is a field of characteristic $\neq$ $2, \phi$ a non-degenerate quadratic form over $F$ (in fact, we even assume that $\phi$ is anisotropic in most places), $X$ the projective quadric $\phi=0, X^{r}$ for any $r \geq 1$ the direct product of $r$ copies of $X, \mathrm{Ch}\left(X^{r}\right)$ the modulo 2 Chow group of $X^{r}$. The reduced Chow group $\overline{\mathrm{Ch}}\left(X^{r}\right)$ is defined as

$$
\overline{\mathrm{Ch}}\left(X^{r}\right)=\operatorname{Im}\left(\mathrm{Ch}\left(X^{r}\right) \rightarrow \operatorname{colim} \mathrm{Ch}\left(X_{E}^{r}\right)\right),
$$

where the colimit is taken over all field extensions $E / F$. We write $\operatorname{Ch}\left(\bar{X}^{r}\right)$ for this colimit and say cycles (on $\left.\bar{X}^{r}\right)$ for its elements. Note that the homomorphism $\mathrm{Ch}\left(X_{E}^{r}\right) \rightarrow \mathrm{Ch}\left(\bar{X}^{r}\right)$ is an isomorphism as far as $\phi$ is completely split over $E$ (it particular, it is so for an algebraic closure of $F$ ).

A cycle on $\bar{X}^{r}$ is said to be rational (or $F$-rational), if it is inside of $\overline{\mathrm{Ch}}\left(X^{r}\right)$. We also refer to the rational cycles on $\bar{X}^{r}$ as to cycles on $X^{r}$. For an extension $E / F$, a cycle on $\bar{X}^{r}$ is said to be $E$-rational, if it is inside of $\overline{C h}\left(X_{E}^{r}\right) \subset \mathrm{Ch}\left(\bar{X}^{r}\right)$. We also refer to the E-rational cycles on $\bar{X}^{r}$ as to cycles on $X_{E}^{r}$.

We set $D=\operatorname{dim}(X)$ and $d=[D / 2]$. A basis of the group $\operatorname{Ch}(\bar{X})$ (as a vector space over $\mathbb{Z} / 2 \mathbb{Z})$ is given by $h^{i}, l_{i}$ with $i=0,1, \ldots, d$, where $h \in \mathrm{Ch}^{1}(\bar{X})$ is the hyperplane section class (which is rational) while $l_{i} \in \mathrm{Ch}_{i}(\bar{X})$ is the class of an $i$-dimensional linear subspace (which is rational if and only if the Witt index of the quadratic form $\phi$ is $>i$, see Lemma 
2.7). For any $r \geq 2$, a basis of the group $\operatorname{Ch}\left(\bar{X}^{r}\right)$ is given by all $r$-fold external products of the elements of the basis of $\operatorname{Ch}(\bar{X})$.

The inner product $h \cdot l_{i}$ for any $i \in[1, d]$ is equal to $l_{i-1}$; besides, $h^{d+1}=0$. The (modulo 2) total cohomological Steenrod operation $S$ on $\operatorname{Ch}(\bar{X})$ is determined by the formulae $S\left(h^{i}\right)=h^{i} \cdot(1+h)^{i}$ and $S\left(l_{i}\right)=l_{i} \cdot(1+h)^{D-i+1}$ (for the proof of the second formula as well as for a calculation of the binomial coefficients modulo 2 see [7]; for construction of the Steenrod operation on the Chow groups of smooth varieties see [1]); since $S$ commutes with the external products, the formulae given also determine $S$ on $\mathrm{Ch}\left(\bar{X}^{r}\right)$ for all $r \geq 2$.

We say that a cycle $\alpha \in \mathrm{Ch}\left(\bar{X}^{r}\right)$ contains a given basis element $\beta$ (and write $\beta \in \alpha$ ), if $\beta$ appears in the decomposition of $\alpha$ into the sum of basis elements. More generally, for two arbitrary cycles $\alpha^{\prime}, \alpha \in \operatorname{Ch}\left(\bar{X}^{r}\right)$, we say that $\alpha$ contains $\alpha^{\prime}$, if every basis elements contained in $\alpha^{\prime}$ is also contained in $\alpha$. According to this, a rational cycle is called minimal, if it is non-zero and does not contain a proper rational subcycle.

Lemma 2.1 ([6, lemma 4.2]). The intersection (still in the above specific sense) of rational cycles is rational (therefore a minimal cycle is contained in every rational cycle "touched" by it; in particular, a minimal cycle containing a given basis element $\beta$ is unique (although may not exist) and coincides (if exists) with the intersection of all rational cycles containing $\beta$ ).

The basis elements of $\mathrm{Ch}\left(\bar{X}^{r}\right)$ which are external products of powers of $h$ are called non-essential (all non-essential basis elements are rational); the remaining basis elements are called essential. A cycle on $\bar{X}^{r}$ is said to be non-essential, if it does not contain any essential basis element. The essence of a cycle $\alpha \in \operatorname{Ch}\left(\bar{X}^{r}\right)$ is the sum of the essential basis elements contained in $\alpha$. Note that the essence of a rational cycle is rational.

We write $\mathfrak{h}$ for the height of $\phi ; \mathfrak{i}_{0}$ for the usual Witt index of $\phi$ (see [8] for the definition); $\mathfrak{i}_{1}, \ldots, \mathfrak{i}_{\mathfrak{h}}$ for the higher Witt indices of $\phi$; and $0 \leq \mathfrak{j}_{0}<\mathfrak{j}_{1}<\cdots<\mathfrak{j}_{\mathfrak{h}}=[\operatorname{dim}(\phi) / 2]$ for the Witt indices of $\phi_{E}$, where $E$ runs over all field extension of $F$ (so that $\mathfrak{j}_{q}=\mathfrak{i}_{0}+\mathfrak{i}_{1}+\cdots+\mathfrak{i}_{q}$ for any $q \in[0, \mathfrak{h}]$; this equality gives a definition of the higher Witt indices).

We write $F_{0}=F \subset F_{1} \subset \cdots \subset F_{\mathfrak{h}}$ for the fields of the generic splitting tower of the quadratic form $\phi$; besides, for $q \in[1, \mathfrak{h}]$, we write $\phi_{q}$ for the anisotropic part of the quadratic form $\phi_{F_{q}}$ and we write $X_{q}$ for the projective quadric of $\phi_{q}$ (the variety $X_{q}$ is defined over the field $F_{q}$ ). For any $q \in[0, \mathfrak{h}]$ and $r \in[1, \mathfrak{h}-q]$ we therefore have $\mathfrak{j}_{q}=\mathfrak{i}_{0}\left(\phi_{F_{q}}\right), \mathfrak{h}\left(\phi_{q}\right)=\mathfrak{h}-q$, and $\mathfrak{i}_{q+r}=\mathfrak{i}_{r}\left(\phi_{q}\right)$. Note that for any $q \in[1, \mathfrak{h}]$, the field $F_{q}$ is the function field $F_{q-1}\left(X_{q-1}\right)$, and this gives an inductive definition of the generic splitting tower of the quadratic form $\phi$.

We recall the available description of the possible values of the first Witt index of the anisotropic quadratic forms of a given dimension which will be used several times in this paper:

Theorem $2.2([5])$. Assume that $\phi$ is anisotropic. Then there exists an integer $n \geq 0$ with $2^{n}<\operatorname{dim}(\phi)$ such that $\mathfrak{i}_{1} \in\left[1,2^{n}\right]$ and $\mathfrak{i}_{1} \equiv \operatorname{dim}(\phi)\left(\bmod 2^{n}\right)$.

Remark 2.3. Theorem 2.2 implies, in particular, that the higher Witt indices of an odddimensional quadratic form are odd. Therefore Theorem 1.1 gives no information in the odd-dimensional case. 
The original proof of the following very important result is given in [4, th. 6.1]. An alternative proof is available in [7] (see also [6, prop. 3.3(8) and $\S 4]$ ):

Theorem 2.4 ([4]). Assume that $\phi$ is anisotropic. If a (rational) cycle on $X^{2}$ contains $h^{0} \times l_{0}$ and does not contain any $h^{i} \times l_{i}$ with $i>0$, then the integer $\operatorname{dim}(\phi)-\mathfrak{i}_{1}$ is a power of 2 .

The following statement is a scion of [9, th. 4.13]:

Proposition 2.5 ([6, lemma 4.23]). Assume that for some $q \in[0, \mathfrak{h}-1]$ there exists a rational cycle containing $h^{i} \times l_{\text {? }}$ with some $i \in\left[\mathfrak{j}_{q}, \mathfrak{j}_{q+1}\right.$ ) (note that the interval is semi-open) and none of $h^{i} \times l_{\text {? }}$ with $i<\mathfrak{j}_{q}$. Then there exists a rational cycle containing $h^{\mathfrak{j}_{q}} \times l_{\mathfrak{j}_{q+1}-1}$ and none of $h^{i} \times l_{\text {? }}$ with $i<\mathfrak{j}_{q}$.

Remark 2.6. The assumption of Proposition 2.5 is always satisfied for $q=0$ : the rational cycle given by the diagonal (computed, e.g., in [6, cor. 3.9]) contains $h^{0} \times l_{0}$.

The following statement is a consequence of the Springer-Satz for quadratic forms (for the Springer-Satz see [8]):

Lemma 2.7 ([6, cor. 2.5]). The group $\overline{\mathrm{Ch}}(X)$ is generated by the elements $l_{i}$ having $i<\mathfrak{i}_{0}(\phi)$ together with all $h^{i}$.

\section{THE UPPER BOUND}

In this $\oint$ we are assuming that $\phi$ is anisotropic.

Proposition 3.1. Let $\alpha$ be the minimal cycle on $X^{2}$ containing the basis element $h^{0} \times l_{0}$. If $\alpha$ also contains $h^{i} \times l_{i}$ with some positive $i$, then such smallest integer $i$ coincides with the Witt index of $\phi$ over some field extension of $F$; more precisely, $i=\mathfrak{j}_{q}$ for some $q \in[1, \mathfrak{h}-1]$.

Proof. Let $i$ be the smallest positive integer satisfying $h^{i} \times l_{i} \in \alpha$. Note that $i \geq \mathfrak{j}_{1}$ (see $[6, \S 4])$. Let $q \in[1, \mathfrak{h}-1]$ be the biggest integer with $\mathfrak{j}_{q} \leq i$. To prove that $\mathfrak{j}_{q}=i$, we assume that $\mathfrak{j}_{q}<i$. Let $\beta$ be the minimal cycle on $X_{F(X)}^{2}$ containing $h^{\mathfrak{j}_{q}} \times l_{\mathfrak{j}_{q+1}-1}$. This cycle exists and does not contain any $h^{j} \times l_{\text {? }}$ with $j<\mathfrak{j}_{q}$ by Proposition 2.5 because of the $F(X)$-rationality of the cycle $\alpha-\left(h^{0} \times l_{0}\right)$. Let $\eta \in \overline{\mathrm{Ch}}\left(X^{3}\right)$ be a preimage of $\beta$ under the pull-back epimorphism $g_{1}^{*}: \overline{\mathrm{Ch}}\left(X^{3}\right) \rightarrow \overline{\mathrm{Ch}}\left(X_{F(X)}^{2}\right)$, where the morphism $g_{1}: X_{F(X)}^{2} \rightarrow X^{3}$ is induced by the generic point of the first factor of $X^{3}$. We consider $\eta$ as a correspondence from $X$ to $X^{2}$ and set $\mu=\eta \circ \alpha$. The cycle $\delta_{12}^{*}(\mu) \in \overline{\mathrm{Ch}}\left(X^{2}\right)$, where $\delta_{12}: X^{2} \rightarrow X^{3}$ is the morphism $x_{1} \times x_{2} \rightarrow x_{1} \times x_{1} \times x_{2}$, contains $h^{\mathfrak{j}_{q}} \times l_{\mathfrak{j}_{q+1}-1}$ and does not contain any $h^{j} \times l_{\text {? }}$ with $j<\mathfrak{j}_{q}$. Therefore the cycle $\delta_{12}^{*}(\mu) \cdot\left(h^{i-\mathfrak{j}_{q}} \times h^{\mathfrak{j}_{q+1}-1-i}\right)$ contains $h^{i} \times l_{i}$ and does not contain $h^{0} \times l_{0}$. By Lemma 2.1, this gives a contradiction with the minimality of $\alpha$.

The following observation is due to A. S. Merkurjev:

Proposition 3.2. Let $n \geq 0$ be an integer such that $\mathfrak{i}_{1}>2^{n}$. Let $\alpha$ be the minimal cycle on $X^{2}$ containing $h^{0} \times l_{\mathfrak{i}_{1}-1}$ (see Remark 2.6). Let $i$ be such that $h^{i}$ is a factor of some basis element contained in $\alpha$. Then $i$ is divisible by $2^{n+1}$. 
Proof. Considerations similar to that of [6, example 4.22] show that $S^{j}(\alpha)=0$ for any $j$ with $0<j<\mathfrak{i}_{1}$. Since $\alpha$ contains $h^{i} \times l_{i+\mathfrak{i}_{1}-1}$ or $l_{i+\mathfrak{i}_{1}-1} \times h^{i}$, it follows that $S^{j}\left(l_{i+\mathfrak{i}_{1}-1}\right)=0$ for such $j$. Since $S^{2^{v_{2}(i)}}\left(l_{i+\mathfrak{i}_{1}-1}\right) \neq 0$ and $2^{v_{2}(i)} \leq 2^{n}<\mathfrak{i}_{1}$ if $v_{2}(i) \leq n$, it follows that $v_{2}(i) \geq n+1$.

The key to the upper bound of Theorem 1.1 is the following result, which is in fact, in some sense, a more precise version of the upper bound part of Theorem 1.1:

Theorem 3.3. Let $\alpha \in \overline{\mathrm{Ch}}\left(X^{2}\right)$ be the minimal cycle containing $h^{0} \times l_{0}$. Assume that $\alpha$ also contains $h^{i} \times l_{i}$ for some $i>0$. Let $q \in[1, \mathfrak{h}-1]$ be such that

$$
\mathfrak{j}_{q} \leq \min \left\{i>0 \mid \alpha \ni h^{i} \times l_{i}\right\}<\mathfrak{j}_{q+1} .
$$

Then $v_{2}\left(\mathfrak{i}_{q+1}\right) \geq v_{2}\left(\mathfrak{i}_{1}\right)$.

Proof. For $n=v_{2}\left(\mathfrak{i}_{1}\right)$, by Theorem 2.2 , the integer $2^{n}$ divides $\operatorname{dim}(\phi)-\mathfrak{i}_{1}$; therefore it divides as well $\operatorname{dim}(\phi)$.

By Proposition 3.1, the minimal positive $i$ with $\alpha \ni h^{i} \times l_{i}$ is equal to $\mathfrak{j}_{q}$; on the other hand, by Proposition 3.2, the integer $i$ is divisible by $2^{n}$. It follows that $2^{n}$ divides $\operatorname{dim}\left(\phi_{q}\right)=\operatorname{dim}(\phi)-2 \mathfrak{j}_{q}$. Now if we assume that $m<n$ for $m=v_{2}\left(\mathfrak{i}_{q+1}\right)$, we get by Theorem 2.2 (applied to $\phi_{q}$ ) that $\mathfrak{i}_{q+1}=\mathfrak{i}_{1}\left(\phi_{q}\right)$ is equal to $2^{m}$ and, in particular, is smaller than $\mathfrak{i}_{1}$, a contradiction with $[9$, th. $7.7(1)]$ (see also $[6, \S 4]$ ).

Proof of the upper bound relation of Theorem 1.1. Clearly, it suffices to prove the upper bound inequality of Theorem 1.1 only for $q=1$. Let $\alpha$ be the minimal cycle on $X^{2}$ containing $h^{0} \times l_{0}$. Since $\operatorname{dim}(\phi)-\mathfrak{i}_{1}$ is not a power of 2 (by the special assumption of the upper bound part of Theorem 1.1), the hypothesis of Theorem 3.3 is satisfied by Theorem 2.4. Consequently, by Theorem $3.3, v_{2}\left(\mathfrak{i}_{1}\right) \leq v_{2}\left(\mathfrak{i}_{q+1}\right)$ for some $q \in[1, \mathfrak{h}-1]$.

\section{A TRICK}

A simple (may be strange looking) idea developed in this $\S$ allows one to avoid a solid amount of direct computation done in $[6, \S 6]$. One can say that the (only real) difference between the proof of Vishik's conjecture given in [6] and the proof via the upper bound of Theorem 1.1 presented here in $\S 6$, is contained in the current $\S$.

Assume for a moment that $\phi$ is isotropic and let $\psi$ be a Witt-equivalent to $\phi$ quadratic form with $\operatorname{dim}(\psi)<\operatorname{dim}(\phi)$. We write $n$ for the integer $(\operatorname{dim}(\phi)-\operatorname{dim}(\psi)) / 2$. Let $Y$ be the projective quadric given by $\psi$.

Let us write $\operatorname{Ch}\left(\bar{X}^{*}\right)$ for the direct sum $\bigoplus \operatorname{Ch}\left(\bar{X}^{r}\right)$ taken over all $r \geq 1$ and consider the commuting with the external products $*$-homogeneous group homomorphisms

$$
p r^{*}: \operatorname{Ch}\left(\bar{X}^{*}\right) \rightarrow \operatorname{Ch}\left(\bar{Y}^{*}\right) \text { and } i n^{*}: \operatorname{Ch}\left(\bar{Y}^{*}\right) \rightarrow \operatorname{Ch}\left(\bar{X}^{*}\right)
$$

determined by $\operatorname{pr}^{1}\left(h^{i}\right)=h^{i-n}, \operatorname{pr}^{1}\left(l_{i}\right)=l_{i-n}, i n^{1}\left(h^{i}\right)=h^{i+n}, i n^{1}\left(l_{i}\right)=l_{i+n}\left(h^{i}\right.$ and $l_{i}$ are (defined as) 0 as far as $i$ is outside of the interval of the admissible values).

Obviously, the composite $p r^{*} \circ i n^{*}$ is the identity. Moreover, both $p r^{*}$ and $i n^{*}$ preserve rationality of cycles (see [6, cor. 2.4]). 
Lemma 4.1. Let $\delta_{X}: X \rightarrow X^{2}$ and $\delta_{Y}: Y \rightarrow Y^{2}$ be the diagonal morphisms. Let $\beta$ be a cycle on $\bar{Y}^{2}$ such that $\beta \not l_{d-n} \times l_{d-n}$ in the case of even $D$. Then

$$
\delta_{X}^{*}\left(i n^{2}(\beta)\right)=h^{n} \cdot i n^{1}\left(\delta_{Y}^{*}(\beta)\right) .
$$

Proof. A direct verification on the basis.

In the following Proposition we do not assume that $\phi$ is isotropic anymore.

Proposition 4.2. Let $\alpha$ be a homogeneous cycle on $\bar{X}^{2}$. Assume that for some $q \in$ $[0, \mathfrak{h}-1]$ the cycle $\alpha$ is $F_{q}$-rational and does not contain any $h^{i} \times l_{\text {? }}$ or $l_{?} \times h^{i}$ with $i<j_{q}$. Then $\delta_{X}^{*}(\alpha)$ is non-essential (in particular, $\delta_{X}^{*}(\alpha)=0$ if $\operatorname{codim} \alpha>d$ ).

Proof. If $\operatorname{codim} \alpha>D$, then the cycle $\delta_{X}^{*}(\alpha)$ is zero simply because its dimension is negative; below we assume that $\operatorname{codim} \alpha \leq D$.

Since $\alpha$ is $F_{q}$-rational, $\alpha \not \supset l_{d} \times l_{d}$ by [6, lemma 4.1]; therefore, $\alpha$ contains none of $l_{i} \times l_{j}$ (those different from $l_{d} \times l_{d}$ are excluded simply by the dimension assumption).

Let $\alpha^{\prime}$ be the essence of $\alpha$ (the definition of essence is given in $\S 2$ ). The cycle $\alpha^{\prime}$ is still $F_{q}$-rational and $\delta_{X}^{*}\left(\alpha^{\prime}\right)$ is the essence of $\delta_{X}^{*}(\alpha)$.

The remaining assumption on $\alpha$ ensures that $\alpha^{\prime}=i n^{2}(\beta)$ for some $\beta \in \operatorname{Ch}\left(\bar{X}_{q}^{2}\right)$. Since $\beta=p r^{2}\left(\alpha^{\prime}\right)$, the cycle $\beta$ is rational (where "rational" means " $F_{q}$-rational" because $F_{q}$ is the field of definition for the quadric $X_{q}$ ) and satisfies the assumption of Lemma 4.1 (with $\left.n=\mathfrak{j}_{q}\right)$. By the formula of Lemma 4.1, it follows that $\delta_{X}^{*}\left(\alpha^{\prime}\right) \in h^{\mathfrak{j}_{q}} \cdot \overline{\mathrm{Ch}}\left(X_{F_{q}}\right)$. The group $h^{\mathrm{j}_{q}} \cdot \overline{\mathrm{Ch}}\left(X_{F_{q}}\right)$ consists of non-essential elements by Lemma 2.7 .

\section{THE LOWER BOUND}

Assume that we are given a counter-example (with $q=1$ and with an anisotropic quadratic form) to the lower bound inequality of Theorem 1.1: an even-dimensional anisotropic quadratic form $\phi$ of height $>1$ with $v_{2}\left(\mathfrak{i}_{1}\right) \leq v_{2}\left(\mathfrak{i}_{2}, \ldots, \mathfrak{i}_{\mathfrak{h}}\right)-2$. Let $n=v_{2}\left(\mathfrak{i}_{1}\right)$. Since $2^{n+2}$ divides $\mathfrak{i}_{q}$ for each $q \in[2, \mathfrak{h}]$, Theorem 2.2 shows that $\mathfrak{i}_{1}$ is a power of 2 , that is, $\mathfrak{i}_{1}=2^{n}$. Note that the difference $\operatorname{dim}(\phi)-\mathfrak{i}_{1}$ can not be a power of 2 (because it is bigger that $2^{n}$ and congruent to $2^{n}$ modulo $2^{n+3}$ ), therefore, by Theorem 2.4 , the minimal cycle $\alpha \in \overline{\operatorname{Ch}}\left(X^{2}\right)$ containing $h^{0} \times l_{0}$ also contains $h^{i} \times l_{i}$ for some $i>0$. Moreover, by Proposition 3.1, $\min \left\{i>0 \mid \alpha \ni h^{i} \times l_{i}\right\}=\mathfrak{j}_{q}$ for some $q \in[1, \mathfrak{h}-1]$.

We fix the following notation (using the particular $q$ introduced above):

$$
\begin{aligned}
a & =\mathfrak{i}_{1}, \\
b & =\mathfrak{i}_{2}+\cdots+\mathfrak{i}_{q}=\mathfrak{j}_{q}-a, \\
c & =\mathfrak{i}_{q+1} .
\end{aligned}
$$

By Lemma 2.1, the following Proposition contradicts the minimality of $\alpha$ and therefore proves that our counter-example is a fake one. The following morphisms are used in the statement: $g_{1}: X_{F(X)}^{2} \rightarrow X^{3}$ is introduced above; $t_{12}: X^{3} \rightarrow X^{3},\left(x_{1}, x_{2}, x_{3}\right) \mapsto\left(x_{2}, x_{1}, x_{3}\right)$ is the transposition of the first two factors of $X^{3} ; \delta_{X^{2}}: X^{2} \rightarrow X^{4},\left(x_{1}, x_{2}\right) \mapsto\left(x_{1}, x_{2}, x_{1}, x_{2}\right)$ is the diagonal morphism of $X^{2}$. Note that by Proposition 2.5 there exists a cycle in $\overline{\mathrm{Ch}}\left(X_{F(X)}^{2}\right)$ containing $h^{a+b} \times l_{a+b+c-1}$. 
Proposition 5.1. Let $\beta \in \overline{\mathrm{Ch}}\left(X_{F(X)}^{2}\right)$ be the minimal cycle containing $h^{a+b} \times l_{a+b+c-1}$. Let $\eta \in \overline{\mathrm{Ch}}\left(X^{3}\right)$ be a preimage of $\beta$ under the pull-back epimorphism $g_{1}^{*}$. Let $\mu$ be the essence of the composite $\eta \circ \alpha$. Then the cycle

$$
\left(h^{0} \times h^{c-a-1}\right) \cdot \delta_{X^{2}}^{*}\left(t_{12}^{*}(\mu) \circ\left(S^{2 a}(\mu) \cdot\left(h^{0} \times h^{0} \times h^{c-a-1}\right)\right)\right) \in \overline{\operatorname{Ch}}\left(X^{2}\right)
$$

contains $h^{a+b} \times l_{a+b}$ and does not contain $h^{0} \times l_{0}$.

Proof. We recall our notation:

$$
\begin{aligned}
a & =\mathfrak{i}_{1}, \\
b & =\mathfrak{i}_{2}+\cdots+\mathfrak{i}_{q}=\mathfrak{j}_{q}-a, \\
c & =\mathfrak{i}_{q+1} .
\end{aligned}
$$

We keep in mind that $a=2^{n}, b \geq 0,2^{n+2}$ divides $b$ and $c$, and $\operatorname{dim}(\phi) \equiv 2 a\left(\bmod 2^{n+2}\right)$ (the congruence is in fact valid even modulo $2^{n+3}$ but we do not care).

Note that for a given $i$, the basis element $h^{i} \times l_{i}$ appears in $\alpha$ only if $i$ is outside of the open interval $(0, a+b)$. Since the cycle $\beta$ does not contain any basis element having $h^{i}$ with $i<a+b$ as a factor and is symmetric (by [6, lemma 4.17]), we have $\beta=\beta_{0}+\beta_{1}$, where

$$
\begin{aligned}
& \beta_{0}=\operatorname{Sym}\left(h^{a+b} \times l_{a+b+c-1}\right) \\
& \beta_{1}=\operatorname{Sym}\left(\sum_{i \in I} h^{i+a+b} \times l_{i+a+b+c-1}\right)
\end{aligned}
$$

with some set of positive integers $I$, where Sym of a cycle on $\bar{X}^{2}$ is the symmetrization, that is, the cycle plus its transpose. Furthermore

$$
\mu \equiv h^{0} \times \beta+h^{a+b} \times \gamma \quad\left(\bmod \left(h^{1+a+b} \times h^{0} \times h^{0}\right) \cdot \operatorname{Ch}\left(\bar{X}^{3}\right)\right)
$$

with $\gamma=\gamma_{0}+\gamma_{1}$, where

$$
\begin{aligned}
& \gamma_{0}=x \cdot\left(h^{0} \times l_{a+b+c-1}\right)+y \cdot\left(l_{a+b+c-1} \times h^{0}\right), \\
& \gamma_{1}=\sum_{j \in J} h^{j} \times l_{j+a+b+c-1}+\sum_{j \in J^{\prime}} l_{j+a+b+c-1} \times h^{j}
\end{aligned}
$$

for some modulo 2 integers $x, y \in \mathbb{Z} / 2 \mathbb{Z}$ and some sets $J, J^{\prime} \subset \mathbb{Z}_{>0}$.

Lemma 5.2. One has: $x=y=1, I \subset \mathbb{Z}_{\geq c}$, and $J, J^{\prime} \subset \mathbb{Z}_{\geq a+b+c}$.

Proof. To determine $y$, consider the cycle $\delta_{13}^{*}(\mu) \cdot\left(h^{0} \times h^{c-1}\right) \in \overline{\mathrm{Ch}}\left(X^{2}\right)$ where $\delta_{13}: X^{2} \rightarrow$ $X^{3}$ is the morphism $\left(x_{1}, x_{2}\right) \mapsto\left(x_{1}, x_{2}, x_{1}\right)$. This rational cycle does not contain $h^{0} \times l_{0}$, while the coefficient of $h^{a+b} \times l_{a+b}$ is equal to $1+y$; consequently, $y=1$ by the minimality of $\alpha$.

Similarly, using $\delta_{12}^{*}$, one shows that $x=1$ (but actually the value of $x$ is not important for our future purpose).

To show that $I \subset \mathbb{Z}_{\geq c}$, assume that $i<c$ for some $i \in I$. Then $l_{i+a+b} \in \overline{\operatorname{Ch}}\left(X_{F_{q+1}}\right)$ and therefore the cycle

$$
l_{i+a+b+c-1}=\left(p r_{3}\right)_{*}\left(\left(l_{0} \times l_{i+a+b} \times h^{0}\right) \cdot \mu\right)
$$


(where $p r_{3}: X^{3} \rightarrow X$ is the projection onto the third factor) is $F_{q+1}$-rational. This is a contradiction with Lemma 2.7 (note that $i>0$ ) because $i+a+b+c-1 \geq a+b+c=$ $\mathfrak{j}_{q+1}(X)=\mathfrak{i}_{0}\left(X_{F_{q+1}}\right)$.

To prove the statement on $J$, let us assume the contrary: there exists $j \in J$ with $j<a+b+c$. Then $l_{j} \in \overline{\operatorname{Ch}}\left(X_{F_{q+1}}\right)$ and therefore

$$
l_{j+a+b+c-1}=\left(p r_{3}\right)_{*}\left(\left(l_{a+b} \times l_{j} \times h^{0}\right) \cdot \mu\right) \in \overline{\mathrm{Ch}}\left(X_{F_{q+1}}\right),
$$

a contradiction (note that $j>0$ ). The statement on $J^{\prime}$ is proved similarly.

Lemma 5.3. The cycle $\beta$ is $F_{1}$-rational. The cycles $\gamma$ and $\gamma_{1}$ are $F_{q+1}$-rational.

Proof. Let $\mathrm{pr}_{23}: X^{3} \rightarrow X^{2},\left(x_{1}, x_{2}, x_{3}\right) \mapsto\left(x_{2}, x_{3}\right)$ be the projection onto the product of the second and the third factors of $X^{3}$. The cycle $l_{0}$ is $F_{1}$-rational, therefore $\beta=$ $\left(p r_{23}\right)_{*}\left(\left(l_{0} \times h^{0} \times h^{0}\right) \cdot \mu\right)$ is $F_{1}$-rational. The cycle $l_{a+b}$ is $F_{q+1}$-rational, therefore $\gamma=$ $\left(p r_{23}\right)_{*}\left(\left(l_{a+b} \times h^{0} \times h^{0}\right) \cdot \mu\right)$ is $F_{q+1}$-rational. Since $\gamma_{0}$ is $F_{q+1}$-rational, it follows that $\gamma_{1}$ $F_{q+1}$-rational as well.

Setting

$$
\xi=\delta_{X^{2}}^{*}\left(t_{12}^{*}(\mu) \circ\left(S^{2 a}(\mu) \cdot\left(h^{0} \times h^{0} \times h^{c-a-1}\right)\right)\right),
$$

we continue the proof of Proposition 5.1 which states that the cycle $\xi \cdot\left(h^{0} \times h^{c-a-1}\right) \in$ $\overline{\mathrm{Ch}}\left(X^{2}\right)$ contains $h^{a+b} \times l_{a+b}$ and does not contain $h^{0} \times l_{0}$.

If the cycle $\xi \cdot\left(h^{0} \times h^{c-a-1}\right)$ contains $h^{0} \times l_{0}$, then $\xi \ni h^{0} \times l_{c-a-1}$. Passing from $F$ to $F_{1}=F(X)$, we get

$$
\overline{\mathrm{Ch}}\left(X_{F(X)}\right) \ni\left(p r_{2}\right)_{*}\left(\left(l_{0} \times h^{0}\right) \cdot \xi\right)=l_{c-a-1}
$$

$\left(p r_{2}: X^{2} \rightarrow X\right.$ is the projection onto the second factor of $\left.X^{2}\right)$, a contradiction with Lemma 2.7 because $c-a-1 \geq a=\mathfrak{i}_{1}(X)=\mathfrak{i}_{0}\left(X_{F(X)}\right)$.

It remains to show that $h^{a+b} \times l_{b+c-1} \in \xi$. Equivalently, it remains to show that

$$
\delta_{X}^{*}\left(\gamma \circ\left(S^{2 a}(\beta) \cdot\left(h^{0} \times h^{c-a-1}\right)\right)+\beta \circ\left(S^{2 a}(\gamma) \cdot\left(h^{0} \times h^{c-a-1}\right)\right)\right)=l_{b+c-1}
$$

with $\delta_{X}: X \rightarrow X^{2}$ being the diagonal morphism of $X$.

We start by showing that

$$
\delta_{X}^{*}\left(\beta \circ\left(S^{2 a}(\gamma) \cdot\left(h^{0} \times h^{c-a-1}\right)\right)\right)=0 .
$$

Note that $S^{2 a}$ vanishes on $h^{0} \times l_{a+b+c-1}$. Therefore $S^{2 a}(\gamma)=S^{2 a}\left(\gamma_{1}\right)$. Also note, that we may assume that $\operatorname{dim}(X) \geq 4(a+b+c)-2$ because otherwise $\gamma_{1}=0$. It is straighforward to see that for any $j<a+b+c$ none of the basis elements $h^{j} \times l_{j+b+c-1}$ and $l_{j+b+c-1} \times h^{j}$ is present in $\beta \circ\left(S^{2 a}\left(\gamma_{1}\right) \cdot\left(h^{0} \times h^{c-a-1}\right)\right.$ ) (look at the index of the first factor of the basis elements contained in $S^{2 a}\left(\gamma_{1}\right)$ and use Lemma 5.2). Taking in account Lemma 5.3, we obtain the relation (1) as a consequence of Proposition 4.2. 
Since $S^{2 a}\left(\beta_{0}\right)=\operatorname{Sym}\left(h^{2 a+b} \times l_{b+c-1}\right)$, we have $\gamma_{0} \circ\left(S^{2 a}\left(\beta_{0}\right) \cdot\left(h^{0} \times h^{c-a-1}\right)\right)=l_{b+c-1} \times h^{0}$ and

$$
\delta_{X}^{*}\left(\gamma_{0} \circ\left(S^{2 a}\left(\beta_{0}\right) \cdot\left(h^{0} \times h^{c-a-1}\right)\right)\right)=l_{b+c-1} .
$$

The composite $\gamma_{0} \circ\left(S^{2 a}\left(\beta_{1}\right) \cdot\left(h^{0} \times h^{c-a-1}\right)\right)$ is 0 by the following reason. Every basis element included in the cycle $S^{2 a}\left(\beta_{1}\right) \cdot\left(h^{0} \times h^{c-a-1}\right)$ has on the second factor place either $l_{j}$ with $j \geq b+c>0$ or $h^{j}$ with $j \geq b+2 c-1>a+b+c-1$ (while the two basis elements of $\gamma_{0}$ have $h^{0}$ and $l_{a+b+c-1}$ on the first factor place). Consequently

$$
\delta_{X}^{*}\left(\gamma_{0} \circ\left(S^{2 a}\left(\beta_{1}\right) \cdot\left(h^{0} \times h^{c-a-1}\right)\right)\right)=0 .
$$

It is straightforward to see that for any $j<a+b+c$ none of the basis elements $h^{j} \times l_{j+b+c-1}$ and $l_{j+b+c-1} \times h^{j}$ is present in $\gamma_{1} \circ\left(S^{2 a}(\beta) \cdot\left(h^{0} \times h^{c-a-1}\right)\right.$ ) (look at the index of the second factor of the basis elements contained in $\left.\gamma_{1}\right)$. Therefore the relation

$$
\delta_{X}^{*}\left(\gamma_{1} \circ\left(S^{2 a}(\beta) \cdot\left(h^{0} \times h^{c-a-1}\right)\right)\right)=0
$$

holds by Proposition 4.2 once again taking in account Lemma 5.3.

Taking the sum of the established relations (1)-(4), we finish the proof of Proposition 5.1 (and also the proof of the lower bound inequality of Theorem 1.1).

\section{HOLES IN $I^{n}$}

Let $W(F)$ be the Witt ring ([8, def. 1.2 of ch.2]) of the classes of the quadratic forms over the field $F$, and let $I(F) \subset W(F)$ be the ideal of the classes of all even-dimensional forms.

Here we show how the lower bound inequality of Theorem 1.1 implies

Theorem $6.1([6])$. Let $n \geq 2$ be an integer, $\phi$ an anisotropic quadratic form such that $\phi \in I(F)^{n}$ and $2^{n}<\operatorname{dim}(\phi)<2^{n+1}$. Then $\operatorname{dim}(\phi)=2^{n+1}-2^{i+1}$ for some $i \in[0, n-2]$.

Proof. Assume that we are given a counter-example $\phi$. We replace $F$ by the biggest field $F_{q}$ of the generic splitting tower of $\phi$ such that the dimension of the anisotropic part of $\phi_{F_{q}}$ is still "wrong", and we replace $\phi$ by this anisotropic part. Applying Theorem 2.2, we see that the situation is as follows: $\operatorname{dim}(\phi)=2^{n+1}-2^{i+1}+2^{j}$ with $i \in[1, n-1]$ and $j \in[1, i-1]$; moreover the higher Witt indices of $\phi$ are $2^{j-1}, 2^{i}, 2^{i+1}, \ldots, 2^{n-1}$. Therefore $\phi$ is a counter-example to the lower bound inequality of Theorem 1.1.

\section{REFERENCES}

[1] Patrick BROSNAN. Steenrod operations in Chow theory. Trans. Amer. Math. Soc. 355 (2003), no. 5, 1869-1903.

[2] Detlev W. HOFFMANN. Isotropy of quadratic forms over the function field of a quadric. Math. Z. 220 (1995), 461-476.

[3] Jürgen HURRELBRINK and Ulf REHMANN. The minimal height of quadratic forms of given dimension. In preparation.

[4] Oleg IZHBOLDIN and Alexander VISHIK. Quadratic forms with absolutely maximal splitting. Contemp. Math. 272 (2000), 103-125.

[5] Nikita A. KARPENKO. On the first Witt index of quadratic forms. Invent. Math. 153 (2003), 455462. 
[6] Nikita A. KARPENKO. Holes in $I^{n}$. Linear Algebraic Groups and Related Structures (Preprint Server) 128 (2003), 1-29.

[7] Nikita A. KARPENKO and Alexander S. MERKURJEV. Rost projectors and Steenrod operations. Doc. Math. 7 (2002), 481-493.

[8] Winfried SCHARLAU. Quadratic and Hermitian Forms. Springer-Verlag, Berlin, Heidelberg, New York, Tokyo, 1985.

[9] Alexander VISHIK. Motives of quadrics with applications to the theory of quadratic forms. Springer Lect. Notes Math. 1835 (2004), 25-101 (Proceedings of the Summer School "Geometric Methods in the Algebraic Theory of Quadratic Forms", Lens, June 2000).

Laboratoire de Mathématiques de Lens, Faculté des Sciences Jean Perrin, Université D’Artois, rue Jean Souvraz SP 18, 62307 Lens Cedex, FRAnCE

Web page: www.math.uni-bielefeld.de/Karpenko

E-mail address: karpenko@euler.univ-artois.fr 